\title{
Review: it is unclear whether treating internalising disorders in childhood prevents substance abuse in adolescence
}

Compton SN, Burns BJ, Egger HL, Robertson E. Review of the evidence base for treatment of childhood psychopathology: internalizing disorders. J Consult Clin Psychol 2002 Dec; 70: 1240-65.

QUESTION: Which psychological, psychopharmacological and adjunctive treatments in childhood have the potential to prevent substance abuse disorders in adolescence?

\section{Design}

Systematic review with narrative synthesis and standardised effect sizes.

\section{Data sources}

The authors searched Medline, PsycInfo and the reference lists of identified articles. Expert reviewers suggested additional studies. The authors identified 18 studies of children with depressive disorders and 33 studies of children with anxiety disorder from the electronic searches.

\section{Study selection}

Controlled studies of treatments for children aged 6-12 years with depressive or anxiety disorders were eligible if they were published in peer reviewed English language journals between 1985-2001.

\section{Data extraction}

The authors did not describe methods for assessing study quality or extracting data.

Source of funding: National Institute on Drug Abuse, Bethesda, Maryland, USA.

For correspondence: S Compton, Duke Child and Family Study Center, Duke University Medical Center, Durham, North

Carolina, USA

scompton@acpub.duke.edu

\section{Main results}

Psychosocial interventions for depression: the authors identified 6 studies of psychosocial interventions for childhood depression. The evidence suggests that cognitivebehavioural therapy and other active interventions may reduce depressive symptoms in children compared with no treatment or wait list controls.

Psychopharmacological interventions for depression: the authors identified 5 randomised trials of antidepressants, 4 of which focused on tricycle antidepressants. Tricyclic antidepressants may be of limited benefit and should not be considered as first line therapy. It was not possible to draw conclusions about selective reuptake inhibitors in childhood depression as only one high quality study was identified.

Psychosocial interventions for anxiety: the authors identified 21 controlled studies of psychosocial interventions for simple phobias, generalised anxiety disorder, separation anxiety disorder, social phobia, post traumatic stress disorder, selective mutism, and refusal to attend school. The evidence suggests that systematic desensitisation, contingency management and cognitive-behavioural therapies are effective for a number of phobias and anxiety disorders. There is less evidence about interventions for post traumatic stress disorder.

Psychopharmacological interventions for anxiety: the authors identified 8 studies on psychopharmacological interventions for obsessive compulsive disorder, separation anxiety, avoidant and overanxious disorder, and other anxiety disorders. Fluoxetine, sertraline and fluvoxamine appear effective for childhood obsessive compulsive disorder. There is less evidence about the effectiveness of medications for other anxiety disorders.

None of the studies reviewed included substance abuse outcomes.

\section{Conclusions}

A number of behavioural, cognitive-behavioural, and pharmacological interventions are associated with reduced childhood depression, phobias, and anxiety disorders. It is unclear whether treating childhood internalising disorders prevents substance abuse in adolescence.

For commentary, please see opposite page. 